\title{
Downlink Rate Distribution in Multi-RAT Heterogeneous Networks
}

\author{
Sarabjot Singh, Harpreet S. Dhillon, and Jeffrey G. Andrews
}

\begin{abstract}
Current wireless networks are becoming increasingly heterogeneous both in terms of the radio access technologies (RATs) and the base station capabilities. Downlink rate or throughput in such heterogeneous networks is a crucial metric and has been primarily studied using system level simulations. To derive the distribution of rate analytically we develop a fairly general tractable model that consists of $M$ different RATs, each deploying up to $K$ different classes of access points (APs), where each class differs in transmit power, path loss exponent, and deployment density. Each class of APs is modeled as an independent Poisson point process (PPP), with mobile user locations modeled as another independent PPP, all channels further consisting of i.i.d. Rayleigh fading. Using a weighted association strategy, the distribution of rate over the entire network is derived. Further, it is shown that in a two-RAT setting there exists an optimum fraction of traffic that should be associated with each RAT to maximize rate coverage, defined as the fraction of users achieving a given rate.
\end{abstract}

\section{INTRODUCTION}

Increasing heterogeneity in current cellular networks is further complicating their mathematical analysis, which is known to be hard even for conventional single-tier networks. Traditional (macro) cellular networks are being overlaid with dense deployments of low-power base stations (pico, femto, relay) in order to eliminate coverage holes and/or boost capacity in the macro-only system [1]. Further, the fast evolving heterogeneous cellular networks (HCNs) are being complemented with the already widely deployed WiFi access points (APs) [2]. Given the exponential growth of wireless traffic [3], wireless networks are expected to become denser and more heterogeneous.

There has been considerable advancement in the theory of HCNs [4]-[6] whereby the location of APs of each tier is assumed to be a homogeneous PPP. The case of modeling macro cellular networks using a PPP has been strengthened through empirical validation in [7] and theoretical validation in [8]. While the PPP assumption offers attractive tractability in modeling interference and hence the signal-to-interferenceand-noise ratio (SINR) in $\mathrm{HCNs}$, the distribution of rate has been elusive. An empirical fitting based approach for association area distribution in a two-tier cellular network and hence rate distribution was proposed in [9]. For general settings, superposition of point processes, each denoting a class of

This work has been supported by the Intel-Cisco Video Aware Wireless Networks (VAWN) program and NSF grant CIF-1016649. The authors are with Wireless Networking and Communications Group (WNCG), The University of Texas at Austin (email: sarabjot@utexas.edu, dhillon@utexas.edu, jandrews@ece.utexas.edu).
APs, leads to the formation of disparate association regions (and hence load distribution) due to differing transmit powers, path loss exponents, and association weights among classes. Thus, resolving to complicated system level simulations for investigating impact of various wireless algorithms on rate, even for preliminary insights, is not uncommon [1], [2]. The goal of this paper is to bridge this gap and provide a tractable framework for deriving the rate distribution in such HetNets.

In this paper, a general $M$-RAT $K$-tier heterogeneous network (HetNet) model is proposed with APs of each tier of each RAT drawn from a homogeneous PPP. This is similar to [4]-[6] with the key difference being the APs of a RAT act as interferers to only the user associated with that RAT. The proposed model is validated by comparing the analytical results with those of a realistic multi-RAT deployment. The main contributions of the paper are summarized below.

Association Regions in HetNet: Distribution of the load or the number of users associated with an AP in HetNets requires characterization of the association regions. Based on the weighted path loss based user association used in this work, the tessellation formed by the association regions of APs (region served by the AP) is identified as a general form of the multiplicatively weighted Poisson Voronoi (PV). Much progress has been made in modeling areas of PV, see [10] and references therein, however that of multiplicatively weighted PV is an open problem. We propose an analytical approximation for characterizing the association areas (and hence the load) of an AP which is shown to be quite accurate in the context of rate distribution.

Rate Distribution in HetNet: We derive the probability that a randomly located user has rate greater than an arbitrary threshold, which is called rate coverage. Equivalently this is the complementary cumulative distribution function (CCDF) of rate over the entire network. Under certain plausible scenarios the derived expression is in closed form. Our analysis shows that in a simplified two-RAT scenario there exists an optimal amount of traffic that should be offloaded from one RAT to another for maximizing rate coverage.

\section{SySTEM MODEL}

The system model in this paper considers up to a $K$-tier deployment of the APs for each of the $M$ RATs. The set of APs belonging to the same RAT operate in the same spectrum and do not interfere with the APs of other RATs. The locations of the APs of the $k^{\text {th }}$ tier of the $m^{\text {th }}$ RAT are modeled as a 2-D homogeneous PPP $\Phi_{m k}$ of density $\lambda_{m k}$. The pair $(m, k)$ 
is used to denote the APs of $k^{\text {th }}$ tier of the $m^{\text {th }}$ RAT. The set of all such pairs with non-zero densities in the network is denoted by $\mathcal{V}=\bigcup_{m=1}^{M} \bigcup_{k \in \mathcal{V}_{m}}(m, k)$ with $\mathcal{V}_{m}$ denoting the set of all the tiers of RAT- $m$, i.e., $\mathcal{V}_{m}=\left\{k: \lambda_{m k} \neq 0\right\}$. Further, the users in the network are assumed to be distributed according to an independent homogeneous PPP $\Phi_{u}$ with density $\lambda_{u}$.

Every AP of $(m, k)$ transmits with the same transmit power $\mathrm{P}_{m k}$ over bandwidth $\mathrm{W}_{m k}$. The downlink desired and interference signals are assumed to experience path loss with a path loss exponent $\alpha_{k}$ for the corresponding tier $k$. The power received at a user from an $\mathrm{AP}$ of $(m, k)$ at a distance $x$ is $\mathrm{P}_{m k} h_{x} x^{-\alpha_{k}}$, where $h_{x}$ is the channel power gain. The random channel gains are Rayleigh distributed with unit average power, i.e., $h \sim \exp (1)$. General fading distributions can also be considered at some loss of tractability [11]. The noise is assumed additive with power $\sigma_{m}^{2}$ corresponding to the $m^{\text {th }}$ RAT, which is in general different for different RATs due to different transmission bandwidths.

\section{A. User Association}

For the analysis that follows, let $Z_{m k}$ denote the distance of the typical user from the nearest AP of $(m, k)$. In this paper, a general association metric is used in which a mobile user is connected to a particular RAT-tier pair $(i, j)$ if

$$
(i, j)=\arg \max _{(m, k) \in \mathcal{V}} \mathrm{T}_{m k} Z_{m k}^{-\alpha_{k}},
$$

where $\mathrm{T}_{m k}$ is the association weight for $(m, k)$ and ties are broken arbitrarily. For notational brevity we define

$$
\hat{\mathrm{T}}_{m k} \triangleq \frac{\mathrm{T}_{m k}}{\mathrm{~T}_{i j}}, \hat{\mathrm{P}}_{m k} \triangleq \frac{\mathrm{P}_{m k}}{\mathrm{P}_{i j}}, \text { and } \hat{\alpha}_{k} \triangleq \frac{\alpha_{k}}{\alpha_{j}},
$$

which respectively characterize the association weight ratio, transmit power ratio, and path loss exponent ratio of a nonserving BS to the serving BS. The association model described above leads to the formation of association areas in the 2-D Euclidean plane as described below.

Definition 1. Association region of an AP is the region of the Euclidean plane in which all users are served by the corresponding AP. Mathematically, the association region of an AP of class $(i, j)$ located at $x$ is

$$
\begin{array}{r}
\mathcal{C}_{x_{i j}}:=\left\{y \in \mathbb{R}^{2}:\|y-x\| \leq\left(\frac{\mathrm{T}_{i j}}{\mathrm{~T}_{m k}}\right)^{1 / \alpha_{j}}\left\|y-Z_{m k}\right\|^{\alpha_{k} / \alpha_{j}}\right. \\
\forall(m, k) \in \mathcal{V}\} . \quad{ }^{(2)}
\end{array}
$$

The random tessellation formed by the collection $\left\{\mathcal{C}_{x_{i j}}\right\}$ of association regions is a general case of the circular Dirichlet tessellation [12]. Fig. 1 shows the association regions with two classes of APs in the network $(\mathcal{V}=\{(1,1) ;(2,3)\}$, say) for two ratios of association weights. The path loss exponent used in the figure is $\alpha_{k} \equiv 4$ (" $\equiv$ " is henceforth used to assign the same value to a parameter for all classes of APs).

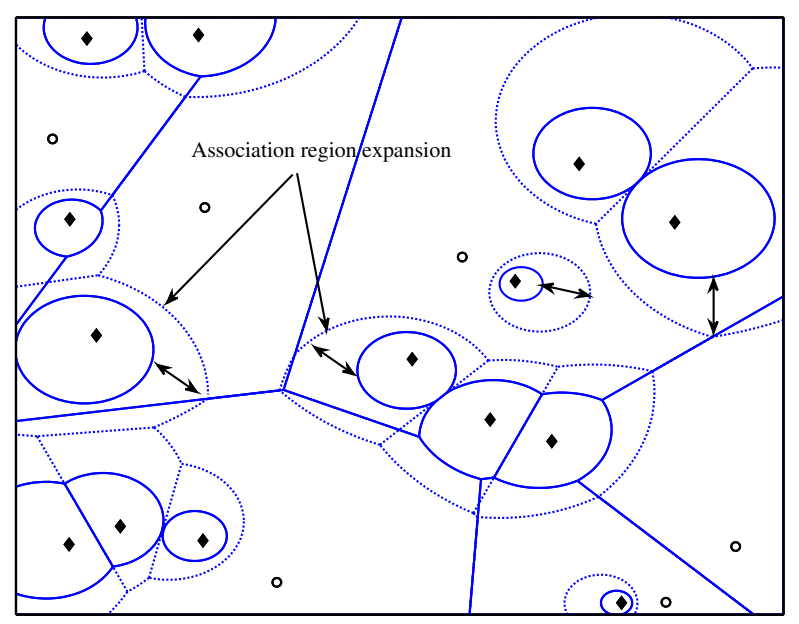

Fig. 1. Association regions of a network with $\mathcal{V}=\{(1,1) ;(2,3)\}$. The APs of $(1,1)$ are shown as hollow circles and those of $(2,3)$ are shown as solid diamonds. Solid lines show the association regions with $\frac{\mathrm{T}_{11}}{\mathrm{~T}_{23}}=20 \mathrm{~dB}$ and dotted lines show the expanded association regions of $(2,3)$ resulting from the use of $\frac{T_{11}}{T_{23}}=10 \mathrm{~dB}$.

\section{B. Resource Allocation}

Proportional rate allocation is assumed, where the rate allocated to each user is proportional to its spectral efficiency. Further, user queues are assumed to be saturated implying that each AP always has data to transmit to its associated mobile users. Thus, the rate of a user associated with $(i, j)$ is

$$
R_{i j}=\frac{\mathrm{W}_{i j}}{N_{i j}} \log \left(1+\operatorname{SINR}_{i j}\right),
$$

where $N_{i j}$ denotes the total number of users served by the AP, henceforth referred to as the load. The presented rate model captures both the congestion effect (through load) and proximity effect (through SINR).

The analysis in this paper is done for a typical user located at the origin. This is allowed by Slivnyak's theorem [13] which states that the properties observed by a typical ${ }^{1}$ point of a PPP, $\Phi$, is same as those observed by a node at origin in the process $\Phi \cup\{0\}$. The SINR of a typical user associated with an AP of $(i, j)$ located at $y$ is

$$
\operatorname{SINR}_{i j}(y)=\frac{\mathrm{P}_{i j} h_{y} y^{-\alpha_{j}}}{\sum_{k \in \mathcal{V}_{i}} I_{i k}+\sigma_{i}^{2}},
$$

where $h_{y}$ is the channel power gain from the tagged AP (AP serving the typical mobile user) located at a distance $y$, $I_{i k}$ denotes the interference from the APs of RAT $-i$ in the tier $k$. The set of APs contributing to interference are from $\bigcup_{k \in \mathcal{V}_{i}} \Phi_{i k} \backslash o$ where $o$ denotes the tagged AP from $(i, j)$. Thus,

$$
I_{i k}=\mathrm{P}_{i k} \sum_{x \in \Phi_{i k} \backslash o} h_{x} x^{-\alpha_{k}} .
$$

\footnotetext{
${ }^{1}$ The term typical and random are interchangeably used in this paper.
} 


\section{Rate Coverage}

This section derives the rate coverage, which can be formally defined as

$$
\mathcal{R}=\mathbb{P}(R>\rho),
$$

and can be equivalently thought of as (i) the probability that a randomly chosen user can achieve a rate threshold $\rho$, (ii) the average fraction of users in the network who at any time achieve rate $\rho$, or (iii) the average fraction of the network area that is receiving rate greater than the rate threshold $\rho$.

\section{A. Load Characterization}

The number of users associated to an AP is dependent on its association area. The following lemma gives the association probability of a typical user to a RAT-tier pair.

Lemma 1. The probability that a typical user is associated with $(i, j)$ is given by

$$
\mathcal{A}_{i j}=2 \pi \lambda_{i j} \int_{0}^{\infty} z \exp \left(-\pi \sum_{(m, k) \in \mathcal{V}} G_{i j}(m, k) z^{2 / \hat{\alpha}_{k}}\right) \mathrm{d} z,
$$

where

$$
G_{i j}(m, k)=\lambda_{m k} \hat{\mathrm{T}}_{m k}^{2 / \alpha_{k}}
$$

If $\alpha_{k} \equiv \alpha$, then the association probability is simplified to

$$
\mathcal{A}_{i j}=\frac{\lambda_{i j}}{\sum_{(m, k) \in \mathcal{V}} G_{i j}(m, k)} .
$$

Proof: The result can be proved by a minor modification of Lemma 1 of [5]. See [14] for the detailed proof.

As observed from the above lemma, the association probability to $(i, j)$ is directly proportional to the corresponding density and association weights. The following two remarks provide an alternate interpretations of the association probability.

Remark 1 . The probability that a typical user is associated with the $i^{\text {th }}$ RAT is given by $\mathcal{A}_{i}=\sum_{j \in \mathcal{V}_{i}} \mathcal{A}_{i j}$. This probability is also the average fraction of the traffic offloaded, referred henceforth as traffic offload fraction, to the $i^{\text {th }}$ RAT.

Remark 2. $\mathcal{A}_{i j}$ is the average fraction of the total area covered by the association regions of the APs of $(i, j)$.

Based on Remark 2 we note that the association area of $(i, j)$ has mean $\frac{\mathcal{A}_{i j}}{\lambda_{i j}}$. Below we propose a linear scaling based approximation for association areas in HetNets which matches the first moment.

Area Approximation: The area $C_{i j}$ of a typical AP of the $j^{\text {th }}$ tier of the $i^{\text {th }}$ RAT can be approximated as

$$
C_{i j}=C\left(\frac{\lambda_{i j}}{\mathcal{A}_{i j}}\right),
$$

where $C(y)$ is the area of a typical cell of PV of density $y$ (a scale parameter).

Remark 3. The approximation is trivially exact for a single tier, single RAT scenario, i.e, for $\|\mathcal{V}\|=1$.
Remark 4. If $\mathrm{T}_{m k} \equiv \mathrm{T}$ and $\alpha_{k} \equiv \alpha$, then the approximation is exact. In this case, $\mathcal{A}_{i j}=\frac{\lambda_{i j}}{\sum_{(m, k) \in \mathcal{V}} \lambda_{m k}}$ and

$$
C\left(\frac{\lambda_{i j}}{\mathcal{A}_{i j}}\right)=C\left(\sum_{(m, k) \in \mathcal{V}} \lambda_{m k}\right) .
$$

With equal association weights and path loss coefficients the HetNet model becomes the superposition of independent PPPs which is again a PPP with density equal to the sum of that of the constituents and hence the resulting tessellation $\left\{\mathcal{C}_{x}\right\}$ is a $\mathrm{PV}$. The right hand side of the above equation is equivalent to a typical association area of a PV with density $\sum_{(m, k) \in \mathcal{V}} \lambda_{m k}$.

Remark 5. Using the distribution proposed in [10] for $C(y)$ the distribution of $C_{i j}$ is

$$
f_{C_{i j}}(c)=\frac{3.5^{3.5}}{\Gamma(3.5)} \frac{\lambda_{i j}}{\mathcal{A}_{i j}}\left(\frac{\lambda_{i j}}{\mathcal{A}_{i j}} c\right)^{2.5} \exp \left(-3.5 \frac{\lambda_{i j}}{\mathcal{A}_{i j}} c\right) .
$$

where $\Gamma(x)=\int_{0}^{\infty} \exp (-t) t^{x-1} \mathrm{~d} t$ is the gamma function. We note that the distribution above involves a two-fold approximation, but, as is shown later, the overall approximation is quite accurate.

To characterize the load at the tagged AP, the implicit area biasing needs to be considered and the probability generating function (PGF) of the other - apart from the typical - users $\left(N_{o, i j}\right)$ associated with the tagged AP need to be characterized.

Lemma 2. The PGF of the other users associated with the tagged AP of $(i, j)$ is

$$
\mathrm{G}_{N_{o, i j}}(z)=3.5^{4.5}\left(3.5+\frac{\lambda_{u} \mathcal{A}_{i j}}{\lambda_{i j}}(1-z)\right)^{-4.5} .
$$

Furthermore, the moments of $N_{o, i j}$ are given by

$$
\mathbb{E}\left[N_{o, i j}^{n}\right]=\sum_{k=1}^{n}\left(\frac{\lambda_{u} \mathcal{A}_{i j}}{\lambda_{i j}}\right)^{k} \mathrm{~S}(n, k) \mathbb{E}\left[C^{k+1}(1)\right],
$$

where $\mathrm{S}(n, k)$ are Stirling numbers of the second kind.

Proof: See Appendix A.

The moments of the typical association region of PV of unit density are available in [15].

\section{B. Main Result}

The rate coverage of a typical user in a generic HetNet setting is stated in the following theorem.

Theorem 1. The rate coverage of a randomly located mobile user in the general HetNet setting of Section II is given by (15) (at the top of next page), where $\rho_{i j}$ is the rate threshold for $(i, j), \hat{\rho}_{i j} \triangleq \rho_{i j} / \mathrm{W}_{i j}, t(x)=$ $2^{x}-1, \quad \mathrm{Z}(a, b, c)=a^{2 / b} \int_{\left(\frac{c}{a}\right)^{2 / b}}^{\infty} \frac{\mathrm{d} u}{1+u^{b / 2}}, D_{i j}\left(k, \tau_{i j}\right)=$ $\hat{\mathrm{P}}_{i k}^{2 / \alpha_{k}} \lambda_{i k} \mathrm{Z}\left(\tau_{i j}, \alpha_{k}, \hat{\mathrm{T}}_{i k} \hat{\mathrm{P}}_{i k}^{-1}\right)$, and $\operatorname{SNR}_{i j}(y)=\frac{\mathrm{P}_{i j} y^{-\alpha_{j}}}{\sigma_{i}^{2}}$.

Proof: See Appendix B

The rate distribution expression for the most general setting requires a single numerical integral and use of lookup tables for $Z$ and $\Gamma$. Since $\mathcal{R}$ decays rapidly for large $n$, the summation 


$$
\begin{aligned}
& \mathcal{R}=\sum_{(i, j) \in \mathcal{V}} 2 \pi \lambda_{i j} \sum_{n \geq 0} \frac{3.5^{3.5}}{n !} \frac{\Gamma(n+4.5)}{\Gamma(3.5)}\left(\frac{\lambda_{u} \mathcal{A}_{i j}}{\lambda_{i j}}\right)^{n} \times\left(3.5+\frac{\lambda_{u} \mathcal{A}_{i j}}{\lambda_{i j}}\right)^{-(n+4.5)} \\
& \quad \times \int_{0}^{\infty} y \exp \left(-\frac{t\left(\hat{\rho}_{i j}(n+1)\right)}{\operatorname{SNR}_{i j}(y)}-\pi\left\{\sum_{k \in \mathcal{V}_{i}} D_{i j}\left(k, t\left(\hat{\rho}_{i j}(n+1)\right)\right) y^{2 / \hat{\alpha}_{k}}+\sum_{(m, k) \in \mathcal{V}} G_{i j}(m, k) y^{2 / \hat{\alpha}_{k}}\right\}\right) \mathrm{d} y, \\
& \overline{\mathcal{R}}=\sum_{(i, j) \in \mathcal{V}} 2 \pi \lambda_{i j} \int_{0}^{\infty} y \exp \left(-\frac{t\left(\hat{\rho}_{i j} \bar{N}_{i j}\right)}{\operatorname{SNR}_{i j}(y)}-\pi\left\{\sum_{k \in \mathcal{V}_{i}} D_{i j}\left(k, t\left(\hat{\rho}_{i j} \bar{N}_{i j}\right)\right) y^{2 / \hat{\alpha}_{k}}+\sum_{(m, k) \in \mathcal{V}} G_{i j}(m, k) y^{2 / \hat{\alpha}_{k}}\right\}\right) \mathrm{d} y,
\end{aligned}
$$

over $n$ in Theorem 1 can be accurately approximated as a finite summation to a sufficiently large value $N_{\max }$. Particularly $N_{\max }=4 \lambda_{u}$ is found to be sufficient in our results presented in Section IV-A.

The rate coverage expression can be further simplified (sacrificing accuracy) if the load at each AP of $(i, j)$ is assumed to equal its mean, which simplifies the rate coverage expression by eliminating the summation over $n$.

Corollary 1. The rate coverage with the mean load approximation is given by (16) (at the top of page) where

$$
\bar{N}_{i j}=\mathbb{E}\left[N_{i j}\right]=1+\frac{1.28 \lambda_{u} \mathcal{A}_{i j}}{\lambda_{i j}} .
$$

Proof: Using an approximation for (26) with $\mathbb{E}_{N_{i j}}\left[\mathcal{S}_{i j}\left(t\left(N_{i j}\right)\right)\right] \approx \mathcal{S}_{i j}\left(t\left(\mathbb{E}\left[N_{i j}\right]\right)\right)$, Lemma 2 gives the first moment of load as $\mathbb{E}\left[N_{i j}\right]=1+\mathbb{E}\left[N_{o, i j}\right]=$ $1+\frac{\lambda_{u} \mathcal{A}_{i j}}{\lambda_{i j}} \mathbb{E}\left[C_{o}^{2}(1)\right]$. Further, using the result that $\mathbb{E}\left[C_{o}^{2}(1)\right]^{\lambda_{j}}=1.28[15]$, the simplified rate coverage expression is obtained.

\section{VAlidation AND RESUlts}

In the discussion that follows we use a specific form of the association weight as $\mathrm{T}_{m k}=\mathrm{P}_{m k} \mathrm{~B}_{m k}$ corresponding to the biased received power based association [1] where $\mathrm{B}_{m k}$ is the association bias for $(m, k)$. In all the results that follow, the transmit powers are $\mathrm{P}_{m 1}=46 \mathrm{dBm}, \mathrm{P}_{m 2}=33 \mathrm{dBm}$ and $\mathrm{P}_{m 3}=23 \mathrm{dBm} \forall m$ and $\mathrm{W}_{m k} \equiv 10 \mathrm{MHz}$. Further, the bias associated with $(1,1)$ is normalized to 1 , or $B_{11}=0 \mathrm{~dB}$.

\section{A. Validation}

In this subsection, the emphasis is on validating the area and mean load approximation (Theorem 1 and Corollary 1 respectively) proposed in the previous sections and on validating the PPP as a suitable AP location model.

1) Analysis: A three-RAT network with $\mathcal{V}=$ $\{(1,1),(2,2),(3,3)\}$ is considered with deployment densities $\lambda_{11}=1 \mathrm{BS} / \mathrm{km}^{2}, \lambda_{22}=3 \mathrm{BS} / \mathrm{km}^{2}, \lambda_{33}=10 \mathrm{BS} / \mathrm{km}^{2}$, user density of $\lambda_{u}=50$ users $/ \mathrm{km}^{2}$, path loss exponents $\alpha_{1}=3.5$, $\alpha_{2}=3.8$, and $\alpha_{3}=4$. Fig. 2 shows the rate distribution obtained through simulation and that from Theorem 1 and Corollary 1 for two sets of association bias values. As can

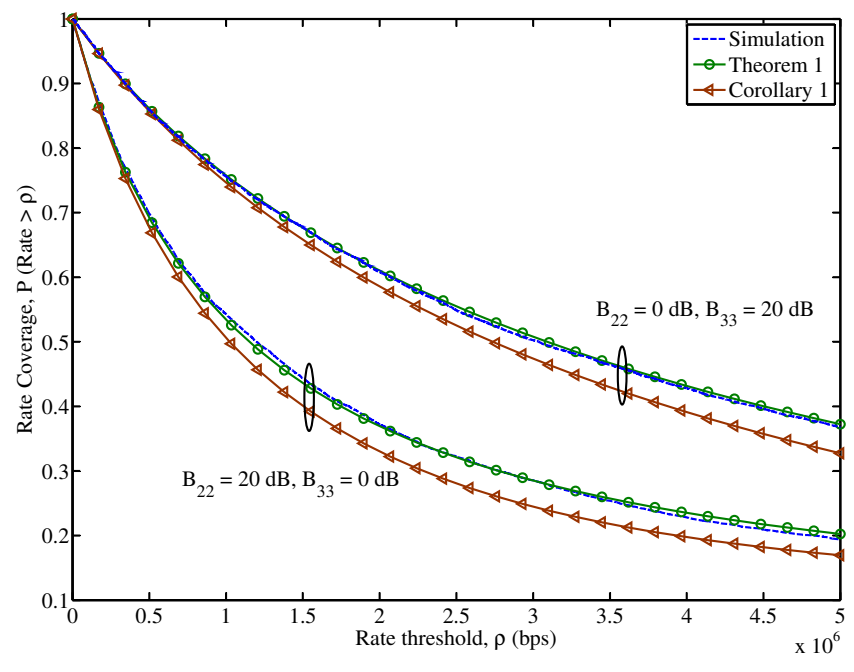

Fig. 2. Rate distribution obtained from simulation, Theorem 1 and Corollary 1 for $\lambda_{22}=3 \lambda_{11}, \lambda_{33}=10 \lambda_{11}$ and $\alpha_{1}=3.5, \alpha_{2}=3.8, \alpha_{3}=4$.

be observed from both the plots, the analytical distributions closely match with the simulated ones and thus validate the analysis of the previous section. Surprisingly, the mean load approximation is not far off from the exact curves.

2) Spatial Location Model: To simulate a realistic two-RAT scenario, the cellular BS location data of a major metropolitan used in [7] is overlaid with that of an actual WiFi deployment [16]. Along with the PPP, a square grid based location model in which the APs for both the RATs are located in square lattice (with different densities) is also used in the following comparison. Denoting the macro tier of cellular BS as $(1,1)$ and WiFi APs as $(2,3), \mathcal{V}=\{(1,1) ;(2,3)\}$ in this setup. The superposition is done such that $\lambda_{23}=10 \lambda_{11}$. Fig. 3 shows the rate distribution of a typical user obtained from the real data along with that of a square grid based model and that from a PPP, i.e., Theorem 1 for two cases. As shown in the plot, Theorem 1 is quite accurate in the context of rate distribution with regards to the actual location data. 


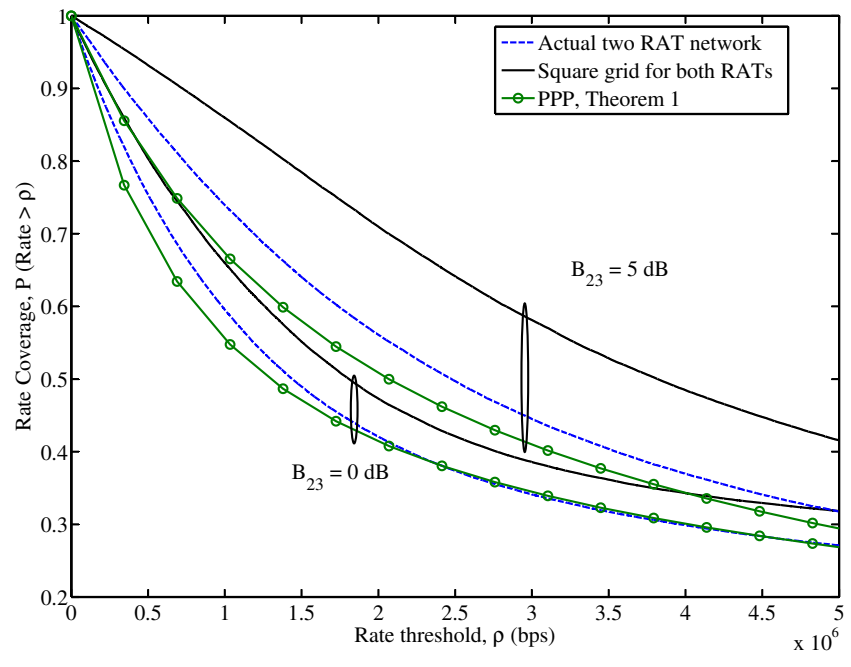

Fig. 3. Rate distribution comparison for the three spatial models: real, grid and PPP for a two-RAT setting with $\lambda_{23}=10 \lambda_{11}$ and $\alpha_{1}=\alpha_{3}=4$.

\section{B. Offloading and Rate Coverage}

Proactive offloading of users to low power APs is important, as load disparity not only leads to sub-optimal rate coverage due to underutilization of resources but also degrades the performance of many applications due to bursty interference caused by the lightly loaded APs [17], [18]. To investigate the impact of offloading on rate we consider a setting of larger tier of RAT-1 overlaid with a low power tier of RAT2 , i.e., $\mathcal{V}=\{(1,1) ;(2,3)\}$. This setting is similar to the widespread use of WiFi APs to offload macrocell traffic. In particular, we investigate the effect of association bias on the $5^{\text {th }}$ percentile rate $\rho_{95}$ with $\mathcal{R}\left(\rho_{95}\right)=0.95$ (i.e., $95 \%$ of the user population receives a rate greater than $\rho_{95}$ ). Variation of the $5^{\text {th }}$ percentile rate with the association bias is shown in Fig. 4 for different densities of RAT-2 APs. In these results, the user density $\lambda_{u}=100 \mathrm{users} / \mathrm{km}^{2}$ and the path loss exponents are $\alpha_{k} \equiv 3.5$. At any particular association bias, as infrastructure density increases, $\rho_{95}$ also increases because of the decrease in load at each AP. However, the optimum association bias for $\rho_{95}$ decreases due to the increase in interference in the corresponding RAT. The optimum association bias and hence the optimal traffic offload fraction can be found by using a linear search in the derived expressions. $\mathrm{T}$

For the presented setting, the optimum traffic offload fraction for different deployment densities is given in Table I. As can be observed, the optimum traffic offload fraction for the second RAT increases with increasing deployment density of the corresponding APs. This is because with increasing density, at the same traffic offload fraction, the load per AP decreases, increasing the affinity of users for the corresponding RAT.

\section{CONClusion}

In this paper we presented a tractable model to derive the rate distribution of a typical user in a $M$-RAT $K$-tier heteroge-
TABLE I

OPTIMUM TRAFFIC OFFLOAD FRACTION

\begin{tabular}{|c|c|c|c|}
\hline & $\lambda_{23}=5 \lambda_{11}$ & $\lambda_{23}=7 \lambda_{11}$ & $\lambda_{23}=10 \lambda_{11}$ \\
\hline $\mathcal{A}_{11}$ & $30.5 \%$ & $29 \%$ & $24.6 \%$ \\
\hline $\mathcal{A}_{23}$ & $69.5 \%$ & $71 \%$ & $75.4 \%$ \\
\hline
\end{tabular}

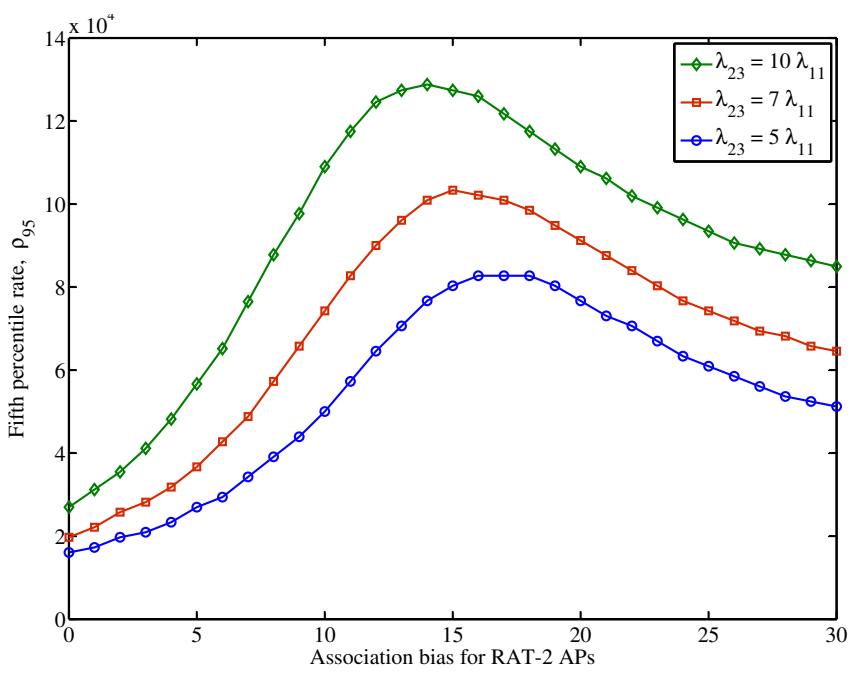

Fig. 4. Effect of association bias for RAT-2 APs on fifth percentile rate.

neous network setting under a flexible association model. It is shown that in a simplified two-RAT setting - similar to cellular and $\mathrm{WiFi}$ - there exists an optimum percentage of the traffic that should be offloaded from one RAT to another to maximize the rate coverage. Insights on inter-tier offload can also be drawn from the presented work. Future work could include modifying the proposed model to derive rate distribution in the presence of joint interference coordination and offloading in heterogeneous cellular networks.

\section{APPENDIX A}

Proof of Lemma 2: As a random user is more likely to lie in a larger association region then in a smaller association region, the distribution of the association area of the tagged $\mathrm{AP}, C_{i j}^{\prime}$, is proportional to its area and can be written as

$$
f_{C_{i j}^{\prime}}(c) \propto c f_{C_{i j}}(c) .
$$

Using the normalization property of the distribution function and (12), the biased area distribution is

$$
\begin{aligned}
f_{C_{i j}^{\prime}}(c) & =\frac{c f_{C_{i j}}(c)}{\mathbb{E}\left[C_{i j}\right]} \\
& =\frac{3.5^{3.5}}{\Gamma(3.5)} \frac{\lambda_{i j}}{\mathcal{A}_{i j}}\left(\frac{\lambda_{i j}}{\mathcal{A}_{i j}} c\right)^{3.5} \exp \left(-3.5 \frac{\lambda_{i j}}{\mathcal{A}_{i j}} c\right) .
\end{aligned}
$$

The location of the other users in the association region of the tagged AP follows the reduced Palm distribution of $\Phi_{u}$ which is the same as the original distribution since $\Phi_{u}$ is a PPP [13, Sec. 4.4]. Thus, using properties of a PPP and (19), the PGF 
of the other users associated with the tagged AP is

$$
\begin{aligned}
\mathrm{G}_{N_{o}, i j}(z) & =\mathbb{E}\left[\exp \left(\lambda_{u} C_{i j}^{\prime}(z-1)\right)\right] \\
& =\int_{c>0} \exp \left(\lambda_{u} c(z-1)\right) f_{C_{i j}^{\prime}}(c) \mathrm{d} c \\
& =3.5^{4.5}\left(3.5+\frac{\lambda_{u} \mathcal{A}_{i j}}{\lambda_{i j}}(1-z)\right)^{-4.5} .
\end{aligned}
$$

Using the PGF, the probability mass function can be derived as

$$
\begin{aligned}
& \mathbb{P}\left(N_{o, i j}=n\right)=\frac{\mathrm{G}_{N_{o, i j}}^{(n)}(0)}{n !} \\
& \quad=\frac{3.5^{3.5}}{n !} \frac{\Gamma(n+4.5)}{\Gamma(3.5)}\left(\frac{\lambda_{u} \mathcal{A}_{i j}}{\lambda_{i j}}\right)^{n} \times\left(3.5+\frac{\lambda_{u} \mathcal{A}_{i j}}{\lambda_{i j}}\right)^{-(n+4.5)} .
\end{aligned}
$$

For the second half of the proof, we use the property that the moments of a Poisson RV, $X \sim \operatorname{Pois}(\lambda)$ (say), can be written in terms of Stirling numbers of the second kind, $\mathrm{S}(n, k)$, as $\mathbb{E}\left[X^{n}\right]=\sum_{k=0}^{n} \lambda^{k} \mathrm{~S}(n, k)$. Now

$$
\begin{aligned}
\mathbb{E}\left[N_{o, i j}^{n}\right] & =\mathbb{E}\left[\mathbb{E}\left[N_{o, i j}^{n} \mid C_{i j}^{\prime}\right]\right] \\
& =\mathbb{E}\left[\sum_{k=0}^{n}\left(\lambda_{u} C_{i j}^{\prime}\right)^{k} \mathrm{~S}(n, k)\right]=\sum_{k=1}^{n} \lambda_{u}^{k} \mathrm{~S}(n, k) \mathbb{E}\left[C_{i j}^{\prime k}\right] .
\end{aligned}
$$

Using (19) and the area approximation (10)

$$
\mathbb{E}\left[C_{i j}^{\prime k}\right]=\frac{\mathbb{E}\left[C_{i j}^{k+1}\right]}{\mathbb{E}\left[C_{i j}\right]}=\frac{\left(\lambda_{i j} / \mathcal{A}_{i j}\right)^{-(k+1)} \mathbb{E}\left[C^{k+1}(1)\right]}{\left(\lambda_{i j} / \mathcal{A}_{i j}\right)^{-1} \mathbb{E}[C(1)]},
$$

and thus

$$
\mathbb{E}\left[N_{o, i j}^{n}\right]=\sum_{k=1}^{n}\left(\frac{\lambda_{u} \mathcal{A}_{i j}}{\lambda_{i j}}\right)^{k} \mathrm{~S}(n, k) \mathbb{E}\left[C^{k+1}(1)\right] .
$$

\section{APPENDIX B}

Proof of Theorem 1: Using (3), the probability that the rate requirement of a user associated with $(i, j)$ is met is

$$
\begin{aligned}
\mathbb{P}\left(R_{i j}>\rho_{i j}\right) & =\mathbb{P}\left(\frac{\mathrm{W}_{i j}}{N_{i j}} \log \left(1+\operatorname{SINR}_{i j}\right)>\rho_{i j}\right) \\
& =\mathbb{P}\left(\operatorname{SINR}_{i j}>2^{\rho_{i j} N_{i j} / \mathrm{W}_{i j}}-1\right) \\
& =\mathbb{E}_{N_{i j}}\left[\mathcal{S}_{i j}\left(t\left(\hat{\rho}_{i j} N_{i j}\right)\right)\right],
\end{aligned}
$$

where $\mathcal{S}_{i j}\left(\tau_{i j}\right) \triangleq \mathbb{E}_{y}\left[\mathbb{P}\left\{\operatorname{SINR}_{i j}(y)>\tau_{i j}\right\}\right], t\left(\hat{\rho}_{i j} N_{i j}\right)=$ $2^{\rho_{i j} N_{i j} / O_{i j}}-1$, and $N_{i j}=1+N_{o, i j}$, i.e., the load at the tagged AP equals the typical user plus the other users. Using Lemma 2, (26) is simplified as

$$
\begin{aligned}
\mathbb{E}_{N_{i j}}\left[\mathcal{S}_{i j}\left(t\left(N_{i j}\right)\right)\right]=\sum_{n \geq 0} \mathbb{P}\left(N_{o, i j}=n\right) \mathcal{S}_{i j}(t(n+1)) \\
=\sum_{n \geq 0} \frac{3.5^{3.5}}{n !} \frac{\Gamma(n+4.5)}{\Gamma(3.5)}\left(\frac{\lambda_{u} \mathcal{A}_{i j}}{\lambda_{i j}}\right)^{n}\left(3.5+\frac{\lambda_{u} \mathcal{A}_{i j}}{\lambda_{i j}}\right)^{-(n+4.5)} \\
\quad \times \mathcal{S}_{i j}\left(t\left(\hat{\rho}_{i j}(n+1)\right)\right)
\end{aligned}
$$

The derivation of $\mathcal{S}_{i j}$ is delegated to [14] due to space constraints. The desired result is obtained by using Lemma 1 along with the fact that $\mathcal{R}=\sum_{(i, j) \in \mathcal{V}} \mathcal{A}_{i j} \mathbb{P}\left(R_{i j}>\rho_{i j}\right)$.

\section{REFERENCES}

[1] A. Damnjanovic, J. Montojo, Y. Wei, T. Ji, T. Luo, M. Vajapeyam, T. Yoo, O. Song, and D. Malladi, "A survey on 3GPP heterogeneous networks," IEEE Wireless Commun. Mag., vol. 18, pp. 10-21, June 2011.

[2] Qualcomm, "A comparison of LTE-Advanced HetNets and WiFi." Whitepaper, available at: http://goo.gl/BFMFR, Sept. 2011.

[3] Cisco, "Cisco Visual Networking Index: Global Mobile Data Traffic Forecast Update, 2011-2016." Whitepaper, available: http://goo.gl/xxLT.

[4] H. S. Dhillon, R. K. Ganti, F. Baccelli, and J. G. Andrews, "Modeling and analysis of $K$-tier downlink heterogeneous cellular networks," IEEE J. Sel. Areas Commun., vol. 30, pp. 550-560, Apr. 2012.

[5] H.-S. Jo, Y. J. Sang, P. Xia, and J. G. Andrews, "Heterogeneous cellular networks with flexible cell association: A comprehensive downlink SINR analysis," IEEE Trans. Wireless Commun., vol. 11, pp. 3484-3495, Oct. 2012.

[6] S. Mukherjee, "Distribution of downlink SINR in heterogeneous cellular networks," IEEE J. Sel. Areas Commun., vol. 30, pp. 575-585, Apr. 2012.

[7] J. G. Andrews, F. Baccelli, and R. K. Ganti, "A tractable approach to coverage and rate in cellular networks," IEEE Trans. Commun., vol. 59, pp. 3122-3134, Nov. 2011

[8] B. Blaszczyszyn, M. K. Karray, and H.-P. Keeler, "Using Poisson processes to model lattice cellular networks," in IEEE INFOCOM, Apr. 2013. Available at: http://arxiv.org/abs/1207.7208.

[9] D. Cao, S. Zhou, and Z. Niu, "Optimal base station density for energyefficient heterogeneous cellular networks," in IEEE International Conf. on Commun., pp. 4379-4383, June 2012.

[10] J.-S. Ferenc and Z. Néda, "On the size distribution of Poisson Voronoi cells," Physica A: Statistical Mechanics and its Applications, vol. 385 , pp. 518 - 526, Nov. 2007.

[11] F. Baccelli, B. Blaszczyszyn, and P. Muhlethaler, "Stochastic analysis of spatial and opportunistic Aloha," IEEE J. Sel. Areas Commun., pp. 11051119, Sept. 2009.

[12] P. F. Ash and E. D. Bolker, "Generalized Dirichlet tessellations," Geometriae Dedicata, vol. 20, pp. 209-243, 1986.

[13] D. Stoyan, W. Kendall, and J. Mecke, Stochastic Geometry and Its Applications. John Wiley \& Sons, 1996.

[14] S. Singh, H. S. Dhillon, and J. G. Andrews, "Offloading in heterogeneous networks: Modeling, analysis, and design insights," IEEE Trans. Wireless Commun., to appear. Available at: http://arxiv.org/abs/1208.1977.

[15] E. N. Gilbert, "Random subdivisions of space into crystals," The Annals of Mathematical Statistics, vol. 33, pp. 958-972, Sept. 1962.

[16] Google, "Google WiFi Mountain View coverage map." Available: http: //wifi.google.com/city/mv/apmap.html.

[17] S. Singh, J. G. Andrews, and G. de Veciana, "Interference shaping for improved quality of experience for real-time video streaming," IEEE $J$. Sel. Areas Commun., vol. 30, pp. 1259-1269, Aug. 2012.

[18] R. Madan, J. Borran, A. Sampath, N. Bhushan, A. Khandekar, and T. Ji, "Cell association and interference coordination in heterogeneous LTE-A cellular networks," IEEE J. Sel. Areas Commun., vol. 28, pp. 1479-1489, Dec. 2010. 